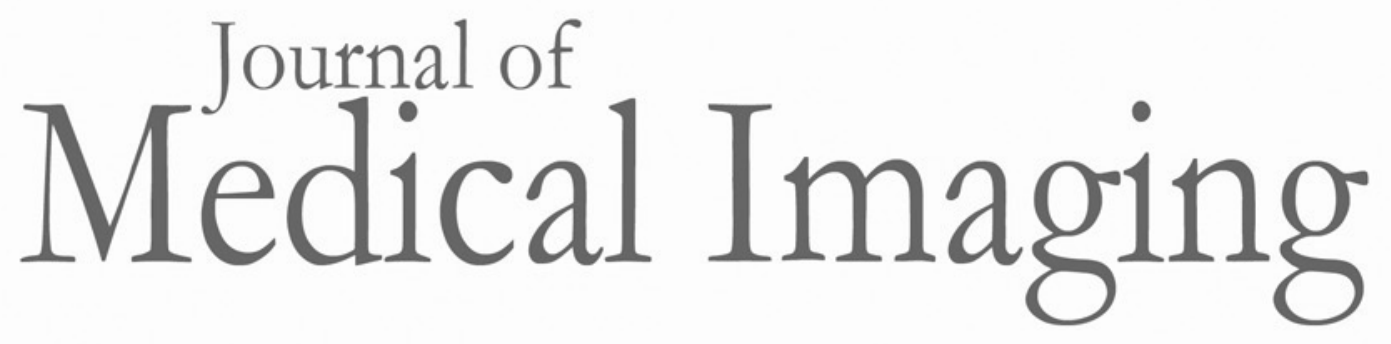

Medicallmaging.SPIEDigitalLibrary.org

\title{
Digital Pathology
}

\author{
Metin N. Gurcan \\ John E. Tomaszewski \\ Anant Madabhushi
}




\section{Digital Pathology}

\author{
Metin N. Gurcan \\ The Ohio State University \\ Department of Biomedical Informatics \\ Columbus, Ohio \\ E-mail: Metin.Gurcan@osumc.edu

\section{John E. Tomaszewski} \\ University at Buffalo \\ The State University of New York \\ Jacobs School of Medicine and Biomedical Sciences \\ Department of Pathology and Anatomical Sciences \\ Buffalo, New York \\ E-mail: johntoma@buffalo.edu
}

\section{Anant Madabhushi}

Case Western Reserve University

Department of Biomedical Engineering

Cleveland, Ohio

E-mail: axm788@case.edu

The special section on digital pathology in the Journal of Medical Imaging is a reflection of the growing research interest in the field and an attempt to showcase the exciting developments in image analytics and machine learning on digitized tissue slides. The call for papers for the special section went out in the spring of 2016, and in response to the call, we received a total of 12 papers. Each of the papers was handled by one of the three guest editors for the special section and was submitted to a rigorous review process involving at least two reviewers. Of the 12 papers, 7 were accepted, reflecting a $67 \%$ acceptance rate.

The paper by J. Xu et al. entitled, "Connecting Markov random fields and active contour models: application to gland segmentation and classification" attempts to converge two fundamental ideas in computer vision-Markov random fields and active contours - for the problem of segmentation of individual glands in digital prostate histopathology images. The authors present an elegant theoretical framework showing the integration of the Markov random field concept of proximity and cliques within active contours. The approach enables invocation of implicit spatial constraints within the deforming contour in order to accurately delineate the gland boundaries. The authors further show that shape-specific features derived from the automatically extracted gland boundaries are capable of discriminating different Gleason grades of prostate cancer from digital pathology images.

The paper by G. Corredor et al. entitled, "Training a cell level classifier for detecting basal cell carcinoma by combining human visual attention maps with low-level handcrafted features" is an attempt to perform classification of skin cancer histopathology images by integrating different classes of image features. The approach involves modeling image features based off the visual attention maps of pathologists looking at the digital pathology images with low-level texture and shape features of individual cells. The approach first involves

C 2017 Society of Photo-Optical Instrumentation Engineers (SPIE) pathologists visually examining pathology images and using a computer program to capture where and how long the human readers look at the images when attempting to identify a possible malignancy. The authors show convincingly that the integration of these visual attention features coupled with lowlevel features results in improved classification performance of the presence or absence of malignancy, compared to the use of low-level image features alone.

J. S. Elkerton et al. present a fully automated machine learning method to differentiate arterioles from venules in mouse histology images in their paper entitled, "Differentiation of arterioles from venules in mouse histology images using machine learning." This differentiation could be useful towards understanding various diseases such as diabetic macular ischemia, hypertensive retinopathy, and Alzheimer's disease. A critical step in developing an automated system is an extensive and comprehensive evaluation. The authors systematically investigate a large set of first- and secondorder and morphological and shape features. Then, they rigorously evaluate the performance of various classifiers through receiver operating characteristic methodology to achieve the best performance in a well-designed set of experiments on high-resolution digital histology images of the mouse hind limb immunostained with smooth muscle $\alpha$-actin.

While most studies focus on large groups of cells in tissues, A. L. Mölder et al.'s study focuses on single cells with noninvasive microscopy. In their paper "Supervised classification of etoposide-treated in vitro adherent cells based on noninvasive imaging morphology," the authors treat adherent cell cultures of DU-145 with a low-concentration anticancer agent and image them for three days using digital holographic microscopy. Morphological changes are observed for different conditions over the time-course by means of image analysis techniques. The authors successfully demonstrate that it is possible to examine long-term processes noninvasively, performing all the measurements in the same culture and detecting changes even before they are detectable by chemical assays. 
This special section also examines the concept that the conversation between pathologists and image scientists requires a mutual understanding of important biological objects. A "prior" in image diagnostics provides a representation starting point for key objects which are hypothesized to subsume critically informative data for a given diagnosis. The definition and use of appropriate priors are part of the art of image diagnostics. At least three types of priors are highlighted in this collection of papers.

F. M. Kahn et al. in their paper "Predicting and replacing the pathological Gleason grade with automated gland ring morphometric features from immunofluorescent prostate cancer images" explore a new structural prior for prostate cancer grading that goes beyond the universally accepted Gleason grading system glandular pattern categories as seen on H\&E brightfield imaging. In this paper, the authors construct gland rings from CK18 cytoplasmic and DAPI nuclear fluorescent labels and interrogate these structures using graph embedding techniques. Gleason patterns 2-5 were effectively modeled in their data set, and this approach also modeled clinical progression and outcomes. The gland ring prior used in this study appears to capture the structural essence of prostate cancer as a deviation from the normal glandular architecture of the prostate when using fluorescence imaging. Just as in Gleason grading, the degree of departure from well-organized gland growth is a marker for aggressiveness.

B. Ginley et al. in their paper "Unsupervised labeling of glomerular boundaries using Gabor filters and statistical testing in renal histology" write a definition of the glomerular tuft boundary as a pipeline of calculations composed of Gabor filtering, Gaussian blurring, statistical F testing, and a distance transform. This pipeline is used to perform rapid unsupervised segmentation of both normal and abnormal glomeruli across multiple standard histochemical stains. This pipeline functions with high sensitivity, specificity, and speed in glomerular segmentation. The starting point or prior in developing this segmentation pipeline is the understanding that the essence of glomerular structure resides in the increased nuclear clustering and textural density, which are different from the sparse extra-glomerular (tubulointerstitial) compartment.

Finally, as a sort of "contextual prior," Platisa et al. in their human observer study "Influence of study design on digital pathology image quality evaluation: the need to define a clinical task" emphasize the clinical task at hand as setting important boundaries on what is judged to be "clinical image quality" (clQ), and how this may be quite different from "perceived image quality" (pIQ). In this paper, the authors explore how these two related but distinct contextual priors provide separate views of what constitutes image quality. Taken together, these three papers highlight the concept that in pathology image analytics you see what you are looking for, and that clearly stated priors are important starting points.

As active researchers in this area, we have observed the enormous growth in digital pathology in the last ten years. Several prominent European medical centers have transitioned to digital pathology, and the first whole slide imaging system has recently been approved for primary reading in the United States. We anticipate the increasing growth in this area to continue in the near future. The papers in this special section provide a glimpse into this exciting future.

Metin N. Gurcan is a professor of biomedical informatics and pathology at The Ohio State University. He received his BSc and PhD degrees from Bilkent University, Ankara, Turkey, and his MSc degree from University of Manchester Institute of Science and Technology, England. His research interests are in medical image analysis and evaluation. $\mathrm{He}$ is a senior member of SPIE and founding co-chair of the SPIE Digital Pathology Conference and Histopathology Image Analysis (HIMA) workshop.

John E. Tomaszewski is a SUNY Distinguished Professor and Chair of Pathology and Anatomical Sciences in the Jacobs School of Medicine and Biomedical Sciences at the University at Buffalo. He received his MD and post graduate training at the University of Pennsylvania where he was a long-term professor. His research interests are in image analytics and integrated diagnostics applied to diagnostic pathology. He serves as co-chair of the SPIE Digital Pathology Conference.

Anant Madabhushi the director of the Center for Computational Imaging and Personalized Diagnostics (CCIPD) and the F. Alex Nason Professor II in the Departments of Biomedical Engineering, Pathology, Radiology, Radiation Oncology, Urology, General Medical Sciences, and Electrical Engineering and Computer Science at Case Western Reserve University. His research interests are in the areas of medical image analysis, machine learning, computational imaging, and digital pathology. 